\title{
La dimension néoplatonicienne du Fondement Ignatien (au contexte du tout des Exercices spirituels de saint Ignace de Loyola)
}

Dieu est un Fondement, un Créateur et un But des créatures. Un chemin à Dieu est le chemin qui conduit de l'état de la disgrâce à l'état de la grâce, c'est-à-dire ici : à l'état de la relation heureuse avec Dieu. Ce chemin conduit par une sanctification et, par conséquent, au perfectionnement chrétien de soi et au rétablissement de l'harmonie dans les relations déformées par le péché originel. Cette pensée est à la base de toute réalité chrétienne. Cette pensée est aussi la base des Exercices spirituels d'Ignace de Loyola, et y compris du Fondement, qui initie les Exercices, ainsi que cette pensée est le principe existentiel, qui est présent, plus ou moins clairement, dans le néoplatonisme chrétien ${ }^{2}$. Cet article est le croquis sur la relation, qui existe entre la théologie du Fondement et le néoplatonisme, mais en rapport avec les Exercices. Le chemin du retour à l'Un devient ici le chemin du retour à Dieu.

\section{La base du Fondement}

Au Fondement il y a le chemin néoplatonicien aux absolus : Bien-Vrai-Beau. Selon Plotin la bonté, la vérité et la beauté sont les attributs de la divinité (chez

${ }^{1}$ Dr. Justyna Sprutta - à la Faculté de Théologie de l'Université Adam Mickiewicz à Poznan chargée de cours de l'histoire d'art et de la protection des monuments et à l'Institut Primatial de la Culture Chrétienne à Bydgoszcz chargée de cours de la théologie dogmatique. Le membre de l'Institut Polonais des Études sur l'Art Mondial et de la Société des Amis des Sciences de Poznań ; e-mail : justynasprutta@o2.pl. ORCID : 0000-0001-9949-9953.

${ }^{2}$ Sur les débuts du néoplatonisme (entre autres sur la théorie des idées de Platon) : cf. M. Gogacz, Szkice o kulturze, Kraków-Warszawa/Struga 1985, p. 36. Sur la pensée platonicienne à la pensée chrétienne chez Pseudo-Denys l'Aréopagite (V et VI siècles); ibidem. 
Pseudo-Denys l'Aréopagite ce sont les noms de Dieu), grâce auxquels Dieu (chez Plotin : l'Un-l'Absolu) permet à l'âme de se faire connaître (Thomas d'Aquin pense la même chose) $)^{3}$. Par exemple, selon Augustin d'Hippone, Dieu est présent en dehors de la réalité créée, mais il entre aussi dans cette réalité, c'està-dire Dieu existe « dans » et « au-dessus », et conduit à cette réalité, à la tête avec l'homme, vers Lui-même et, comme la Bonté-Vérité-Beauté manifeste sa magnificence et sa relation ontologique et agapétologique avec les créatures, par exemple, par le bien des actes initiés par la synergeia ou par le beau de la flore (dans l'Évangile le Créateur lui-même est charmé par son œuvre : le beau des lis champêtres) ${ }^{4}$. Par conséquent, dans le christianisme la triade néoplatonicienne Bien-Vrai-Beau s'identifie avec Dieu, et le chemin vers Lui est le fondement et le but de l'homme, également par les créatures, mais seulement par ces créatures, qui mènent sûrement à Dieu et ne s'éloignent pas de Lui'.

J. Augustyn écrit que le Fondement est « la description concise de la vérité suprême de la foi, qui dit que dans l'existence humaine, Dieu est le but fondamental et le sens de la vie, auquel [c'est-à-dire à Dieu - J.S.] le tout devrait être subordonné $»^{6}$. Le Fondement est d'ailleurs la synthèse et le résumé des quatre semaines, et pas seulement leur début. Il est « la base théologique, éthique, anthropologique et spirituelle des Exercices spirituels de saint Ignace $»^{7}$. Ainsi donc le Fondement suggère le chemin spécifique de connaître Dieu et en même temps

${ }^{3}$ R. Scruton, Piękno, thum. S. Krawczyk, A. Rejniak-Majewska, Łódź 2018, p. 15. Selon Thomas d'Aquin, la vérité, la bonté (il identifie la bonté et la beauté) et l'unité, c'est-à-dire transcendentalia, sont dans toutes les choses, car elles sont les aspects de l'être, c'est-à-dire elles sont les moyens « de la révélation du plus grand don de l'être à l'intellection». Ibidem, p. 16. Encore plus tôt, par exemple, Plotin identifie la bonté et la beauté. Cf. A. Kijewska, Neoplatonizm Jana Szkota Eriugeny. Podmiotowe warunki doświadczenia mistycznego $w$ tradycji neoplatońskiej, Lublin 1994, p. 60.

${ }^{4}$ Cf. P. Kunzmann, Metamorfozy neoplatonizmu, « Poznańskie Studia Teologiczne » vol. XXII (2008), pp. 203-214, ici : p. 207.

${ }^{5}$ Selon Pseudo-Denys l'Aréopagite, l'âme s'approche de Dieu par l'extase. Elle le fait par son mouvement. C'est le mouvement circulaire qui exprime la beauté. Ce mouvement manifeste l'appartenance de l'âme à l'éternité. Il est également le mouvement rectiligne, qui exprime la bonté : ce mouvement dirige l'âme vers les plus parfaits être, et il est le mouvement spiral qui exprime la beauté. Grâce à ce mouvement, l'âme est la même avec elle-même. P. Tendera, Od filozofii światta do sztuki światta, Kraków 2014, p. 89. Selon Philon d'Alexandrie, l'extase est la grâce de Dieu. Plotin comprend la grâce comme possibile grâce aux forces de la nature. G. Reale, Historia filozofii starożytnej. Szkoły epoki Cesarstwa, thum. E.I. Zieliński, Lublin 1999, p. 602.

6 J. Augustyn, ,Ćwiczenia duchowne” $w$ praktyce. Uwagi odnoszace się do udzielania i odprawiania „Ćwiczeń duchownych" św. Ignacego Loyoli z petnym milczeniem i indywidualnym prowadzeniem, Kraków 2013, p. 17. Cf. Ignacy Loyola, Ćwiczenia duchowne, tłum. J. Ożóg, Kraków 1997, 23 (trad. J. Sprutta).

${ }^{7}$ K. Frejusz, Integralny wymiar pedagogii „Ćwiczeń duchownych” św. Ignacego Loyoli, « Studia Bobolanum» 28 (2017), 2, pp. 33-59, ici : p. 43 (trad. J. Sprutta). 
le but de la vie humaine. L'union avec Dieu (qui est mystique c'est-à-dire réaliste aussi) est ce but. Selon S.T. Zarzycki, l'expérience mystique de Dieu est la base des Exercices spirituels (leur première étape est le Fondement), grâce auxquels toute connaissance, à laquelle Ignace " encourage » déjà dans le Fondement), « devient un élément d'une nouvelle synthèse ${ }^{8}$. Cet expérience mystique est aussi réelle et embrasse tout être humain.

Dieu - le Créateur aimant - est « le contenu central » du Fondement, et les créatures sont « le fruit » de la joie, du pouvoir et de l'amour de Dieu. De plus, Dieu est vu comme le Donneur de la vie, qui donne ses biens à l'homme'. Le Fondement ouvre pour ce Dieu et mène à Lui. Déjà le Fondement est le moment de l'expérience approfondie de l'amour de Dieu. Tout le processus des Exercices est couronné par « la contemplation pour obtenir l'amour ». Cette contemplation rend et approfondit cet amour dans l'attitude contemplative. K. Frejusz écrit :

la contemplation pour obtenir l'amour est le point culminant et le couronnement de toute la dynamique des Exercices spirituels de saint Ignace de Loyola. Comme le Fondement, qui est la porte qui ouvre tout le processus (de la croissance), qui a le lieu au temps des quatre semaines des Exercices, 'la contemplation pour obtenir l'amour' synthétise et unie tout le processus ${ }^{10}$.

Cette contemplation est « le pont» spécifique entre le temps de la prière intensive et la vie quotidienne. Ce temps aide au recueillant encore plus se donner à Dieu pour la disposition. Cette contemplation apprend de trouver de Dieu en toutes choses.

Le chemin spirituel, continué par les Semaines de la retraite ignatienne, conduit par les étapes : la purification, l'illumination et l'union. Pseudo-Denys l'Aréopagite suggère ces étapes dans son traité intitulé Théologie mystique ${ }^{11}$. Au tournant du Ve et VIe siècle Pseudo-Denys l'Aréopagite développe la doctrine de ces trois voies du développement spirituel, montrant la triple action de Dieu pour l'âme, c'est-à-dire par la purification et l'illumination, afin que l'union devienne possible. Le premier chemin conduit à la paix de l'âme, le second chemin à la vérité et le tiers chemin à l'amour ${ }^{12}$. Dans Exercices spirituels la $1^{\mathrm{e}}$ Semaine est

${ }^{8}$ S.T. Zarzycki, Ćwiczenia duchowne [dans :] Leksykon duchowości katolickiej, red. M. Chmielewski, Lublin-Kraków 2002, pp. 179-182, ici : pp. 179-180 (trad. J. Sprutta).

${ }^{9}$ W. Królikowski, Ignacjański Fundament. Sztuka korzystania ze stworzeń [dans :] Droga duchowego uporządkowania i miłości. Podstawowe pojęcia w duchowości ignacjańskiej, red. W. Królikowski, Kraków 2017, p. 89.

${ }^{10}$ K. Frejusz, Integralny wymiar pedagogii..., p. 53 (trad. J. Sprutta).

${ }^{11}$ Cf. M. Manikowski, Warunki doświadczenia mistycznego wedtug Pseudo-Dionizego Areopagity. Ujęcie filozoficzne, « Filozofia Chrześcijańska » 15 (2018), 3, pp. 9-29, ici : p. 16.

12 K. Sztuka, Wyobraźnia a rozwój duchowy, Kraków 2010, pp. 220-221. 
le chemin spirituel de purification, $2^{\mathrm{e}}$ Semaine est le chemin spirituel d'illumination, $3^{\mathrm{e}}$ Semaine est le chemin spirituel de renforcement (mais, per exemple, selon E. Przywara cette Semaine est le temps de l'union), et $4^{\mathrm{e}}$ Semaine est le moment d'union ${ }^{13}$. Ignace n'écrit que sur la purification et l'illumination. Il a montré son chemin spirituel par ces étapes dans Le récit du pélerin ${ }^{14}$.

Selon $\mathrm{P}$. Tendera, ces étapes embrassent la réflexion (et la prière) sur les mystères de la vie du Christ, Dieu agit grâce à ses attributs, c'est-à-dire grâce à : la purification, l'illumination et l'union, où la purification correspond au Bien, l'illumination correspond au Vrai et l'union correspond au Beau, parce que l'unité est le principe fondamental de chaque de ces transcendantaux ${ }^{15}$. Ces chemins engagent les trois pouvoirs fondamentaux de l'homme (avec le rôle dominant de l'un de ces pouvoirs à l'étape concrète) : la volonté (le chemin de la purification), la raison/l'intellect (le chemin de l'illumination) et les émotions (le chemin de l'union). Chez Plotin ces trois étapes sont les phases de l'ascension de l'âme vers l'Absolu ${ }^{16}$.

Ignace n'est pas entré directement en contact avec le néoplatonisme, mais grâce à la lecture des écrits de mystiques chrétiens, qui représentaient les courants : dominicain et franciscain ${ }^{17}$. Pour Ignace, la purification, l'illumination et l'union ont leur le lieu dans l'Église qui est le Corps visible du Jésus-Christ ${ }^{18}$.

\section{Le chemin vers le Bien (l'étape de la purification)}

En ce qui concerne la première étape, le néoplatonicien Jean Scot Érigène affirme que la purification de l'âme du mal moral et sa renaissance par l'acquisition de

${ }^{13}$ S. Mrozek, Rekolekcje w domu ze św. Ignacym Loyola, Kraków 2012.

${ }^{14}$ Ignacy Loyola, Pisma wybrane, vol. I, tłum. i oprac. M. Bednarz, Kraków 1968, p. 642.

15 P. Tendera, Od filozofii światta ..., p. 80.

16 Selon Plotin, la première étape est l'étape de l'élimination de l'influence négative du corps (de la matière) sur l'âme. L'âme se compose d'une partie inférieure et supérieure. La partie inférieure est reliée au corps, et la partie supérieure est proche de l'Esprit. Par conséquent, la partie inférieure doit être purifiée (catharsis). Grâce à cette purification, l'âme s'élève à un niveau permettant la réalisation de la vertu (elle n'est pas soumise au corps), et puis elle passe à la contemplation de l'Esprit. La contemplation signifie ici l'étape de l'illumination avec la lumière de l'Esprit. Dans la dernière phase il y a une union avec l'Un, c'est-à-dire l'extase (unio mystica). J. Disse, Metafizyka. Od Platona do Hegla, tłum. A. Węgrzecki, L. Kusak, Kraków 2005, p. 111.

${ }^{17}$ L'expérience spirituelle personnelle de Ignace se lie avec Manresa. C'est la plus importante source. Les autres sources sont : le contact avec la spiritualité franciscaine (cultivé dans le couvent des Clarisses à Arévalo en Espagne), Vita Christi de Ludolfa de Saxe, Imitatio Christi de Thomas à Kempis et Flos Sanctorum de Alons de Villegas. En lisant Imitatio Christi et Flos sanctorum Ignace de Loyola rencontra une terminologie qui est en rapport avec l'esprit du néoplatonisme chrétien.

${ }_{18}$ C'est aussi la vue de saint Basile. Ignace de Loyola est également inspiré surtout par les saints : Bernardin de Sienne, Catherine de Sienne, Ignace d'Antioche, Jean Cassian, Benoît. 
vertus préparent l'homme à la transition vers les étapes suivantes du développement spirituel, c'est-à-dire vers les étapes de la connaissance et de la contemplation (la phase de l'illumination et la phase de l'union) ${ }^{19}$. Auparavant, Platon présente l'opinion que la purification de l'âme détermine le passage de l'obscurité à la lumière, et ce passage permet d'atteindre l'état de bonheur ${ }^{20}$. En conséquence, « l'âme qui a décidé de changer sa vie, devient, à la fin du chemin, remplie de la lumière de la vérité émanant de la profondeur de l'être $\aleph^{21}$; elle est déjà entrée en la phase de l'illumination. Pseudo-Denys l'Aréopagite, écrivant sur la phase de la purification, souligne qu'il faut — quasi dans l'esprit phénoménologique — rejeter tout ce qui est vu et accepté par l'intellect, les êtres et non-êtres, l'activité non intellectuelle et la perception sensuelle pour s'élever, dans l'ignorance, vers Dieu.

Cependant, le chemin ignatien ne requiert pas l'ignorance comme la condition pour créer une relation étroite entre l'homme et Dieu. De plus, la purification (sur le chemin ignatien) n'est pas le processus autonome, qui a la source dans la contemplation du Bien, mais elle a le caractère relationnel et indique l'activité du pouvoir de Jésus-Christ, le Dieu incarné. Le motif lumineux déjà mentionné est également présent sur le chemin ignatien. Il conditionne la purification, en commençant de l'initiateur de ce chemin :

devant l'inondation encore 'aveugle' l'âme [de saint Ignace - J.S.] par les torrents de la lumière divine, Dieu devait d'abord la purifier, montrant à son âme qu'elle ne pouvait rien faire par elle-même, et également, comme elle dépend de Dieu et comment elle devait attribuer tout le bien à Dieu, sans céder aux insinuations de l'amour égoïste ${ }^{22}$.

La condition suggérée par Pseudo-Denys l'Aréopagite comme nécessaire et identifiée à être le « vase vide » situe l'homme au seuil de l'expérience mystique ; après le passage de ce seuil l'homme s'unit avec ce qui est divin ${ }^{23}$. Ce vase sera rempli de la lumière. Déjà Plotin dit que l'âme doit se détourner des choses visibles et se tourner vers la source de la lumière. Le but de sa perception est de voir la lumière qui rend la connaissance. La connaissance et la pensée sont interprètées par ce philosophe comme une façon, tandis que la lumière de l'illumination de Dieu également comme l'objet de la vue ${ }^{24}$.

${ }^{19}$ A. Kijewska, Eriugena, Warszawa 2005, p. 114.

20 J. Skrzypek-Faluszczak, Ocalenie od zła w filozofii Platona, Kraków 2010, p. 213.

${ }^{21}$ Ibidem, p. 217 (trad. J. Sprutta).

22 Przedmowa [dans :] Dziennik św. Ignacego, tłum. M. Bednarz, vol. I, Kraków 1968, p. 287 (trad. J. Sprutta).

${ }^{23}$ M. Manikowski, Warunki doświadczenia mistycznego..., p. 19.

${ }^{24}$ A. Kijewska, Neoplatonizm Jana Szkota Eriugeny..., p. 62. Cf. M.R. Lootens, Augustyn [dans :] Duchowe zmysty. Percepcja Boga w zachodnim chrześcijaństwie, red. P.L. Gavrilyuk, 
La phase de la purification (via purgativa) peut être attribuée au bien comme entre autres l'un des transcendentaux. Également par le bien (l'attitude morale, la dimension éthique de l'existence, l'idée du bien qui se manifeste dans la pensée, la parole, l'acte) l'homme atteint le Bien absolu. Le bien implique l'abandon de l'attitude pécheresse, qui implique la purification spirituelle, et puis la persistance dans l'état de l'esprit purifié. Sur via purgativa, si populaire dans la vie spirituelle du Moyen Âge, engageant le « pensée » discursif et la volonté active, l'homme éloigne les péchés de lui-même (en particulier les péchés lourds), c'est-à-dire comme les péchés éloignent, "par leur nature », de Dieu ${ }^{25}$. La confession générale est le point important à cette phase : de $1^{\mathrm{e}}$ Semaine de la retraite ignatienne.

Une décision de se tourner vers Dieu (l'attitude basée sur la confiance embrasse tout « le moi » humain) devient la décision qui permet de rester dans le bien et, sur ce chemin de la grâce, d'approcher de Dieu, d'approcher couronné dans les Exercices spirituels par la rencontre avec le Verbe Incarné ressuscité. Déjà le Fondement suggère le retour actif à Dieu, c'est-à-dire, comme l'écrit M. Bednarz, « à la source de la paix et du bonheur à travers de la lutte et le service, par le don de soi dans la lutte et le service $»^{26}$. N'oublions pas aussi, que le motif de la lutte et du service a été implanté (à Manresa) dans les Exercices spirituels par le ex-chevalier ${ }^{27}$. Tout processus « d'exercice», initié par le Fondement, à la recherche de la glorification de Dieu et la service à Dieu comme le but ${ }^{28}$.

Être bon et aspirer au Bon absolu comme le but ultime signifie le perfectionnement permanent et, par conséquent, la réalisation de la sainteté, c'est-à-dire de la perfection chrétienne. Selon W. Tatarkiewicz, Platon et des stoïciens ont fait de « la perfection » un terme philosophique, et puis la pensée philosophique chrétienne a transformé ce terme en religieux ${ }^{29}$. Dans le christianisme, le bien qui est à la base de la sainteté, se situe au-dessus de la vérité et de la beauté. Pour Pseudo-Denys l'Aréopagite le bien a une valeur plus haute que le vrai et le beau. Le plus important pour Pseudo-Denys l'Aréopagite est le Bon qui est le nom propre de Dieu. Ce Bon est la première théophanie aussi ${ }^{30}$. T. Stępień dit : le bon est aussi ce qui, comme le premier, vient de Dieu, mais dans l'acte de création s'identifie au

S. Coakley, tłum. A. Gomola, Kraków 2014, pp. 81-98, ici : p. 88.

${ }_{25}$ F. Jalics, Kontemplacja. Wprowadzenie do modlitwy uważności, thum. J. Bolewski, Kraków 2017, p. 16.

${ }^{26}$ M. Bednarz, Całościowa wizja „Ćwiczeń duchownych” św. Ignacego [dans :] Co zabrać ze sobq? Po Fundamencie „Ćwiczeń duchownych”, red. J. Augustyn, Kraków 2012, pp. 13-28, ici : p. 15 (trad. J. Sprutta).

${ }^{27}$ À propos de la genèse et de la signification des Exercices spirituels de saint Ignace de Loyola : cf. S.T. Zarzycki, Ćwiczenia ..., p. 179. Cf. J. Misiurek, Zarys historii duchowości chrześcijańskiej, Lublin 1992, p. 61.

${ }^{28}$ J. Augustyn, ,Ćwiczenia duchowne” w praktyce..., p. 18.

${ }^{29}$ W. Tatarkiewicz, O doskonałości, Lublin 1991, p. 34.

${ }^{30}$ P. Tendera, Od filozofii światta ..., p. 79. 
beau $^{31}$. Pseudo-Denys l'Aréopagite écrit dans son traité intitulé Noms de Dieu: « en effet, la bonté et la beauté sont les mêmes [...]» $»^{32}$.

L'amour est la plus haute et la plus parfaite expression de cette bonté. Dieu est le modèle de l'amour. Son amour charité s'exprime le plus abondamment dans l'acte du salut et dans ses fruits ${ }^{33}$. Cependant, déjà l'acte même de la création est le premier geste de Dieu. Ce geste a sa volonté aimante et libre chez son fondement ${ }^{34}$. K. O'Brien rappelle, que la plus importante vérité est la base des Exercices: Dieu aime inconditionnellement un homme qui (malgré ses limites) a une valeur exceptionnelle et une dignité exceptionnelle aux yeux du Créateur.

La plus naturelle réponse de l'homme à cet amour parfait et à cette fidélité de Dieu dans l'amour pour le pécheur est aussi l'amour inconditionnel : l'homme pour Dieu et pour les autres hommes, et le fait de marquer du respect pour les dons de Dieu. L'amour du Créateur pour les créatures suggère la kénose permanente de Dieu, visible particulièrement dans le sacrement de l'Eucharistie ${ }^{35}$. La réponse et le service mentionnés (l'amour est, entre autres, la base et « le moteur » pour ce service) devraient être basés sur le principe ignatien magis (« meilleur », « plus »). La relation avec Dieu est basée sur l'amour, c'est-à-dire sur l'acceptation de cet amour, qui est le don de Dieu et qui s'exprime dans les dons nombreux et dans la réponse mentionnée. Par conséquent, la relation avec Dieu est basée sur la louange de Dieu, le honorer rendu et le service, Le montrant comme le but de la vie humaine. La dernière étape du chemin spirituel présente cette relation dans la dimension mystique ou presque mystique ${ }^{36}$. Le Fondement rappelle que la recherche de plus grand bien non pour la propre gloire, mais pour plus grande gloire de Dieu, est le plus important de cette attitude. L'homme doit s'ouvrir à la grâce du salut et accepter cette grâce, incorporant le principe magis dans la vie quotidienne.

L'atteindre à Dieu est l'atteindre à la Vérité absolue (par la connaissance de Dieu, c'est-à-dire la connaissance de la vérité sur Lui), à la Bonté absolue (par le perfectionnement, c'est-à-dire l'atteindre de la sainteté, qui s'exprime le plus parfaitement dans l'amour pour Dieu, pour les autres hommes et également dans l'amour pour des autres créatures) et à la Beauté absolue (par l'extase de Dieu) ;

31 T. Stępień, Pseudo-Dionizy Areopagita. Chrześcijanin i platonik, Warszawa 2010, p. 104.

32 Pseudo-Dionizy Areopagita, Pisma teologiczne, thum. M. Dzielska, Kraków 2005, IV, 7 (trad. J. Sprutta).

${ }^{33}$ Cf. J. Augustyn, „Ćwiczenia duchowne” w praktyce..., p. 18.

${ }^{34}$ Cf. J. Strumiłowski, Neoplatońska idea wyjścia i powrotu jako strukturalna zasada wyrażania prawd teologicznych i jej dekonstrukcyjna reinterpretacja, « Teologia w Polsce » 10 (2016), 2, pp. 129-140, ici : p. 131.

${ }^{35}$ K. O’Brien, „Ćwiczenia duchowe”. Przewodnik po przygodzie życia, thum. G. Gomola, A. Gomola, Kraków 2016, p. 55.

${ }^{36}$ Cf. W. Królikowski, Ignacjański Fundament ..., p. 85. 
cette extase trouve son expression dans la contemplation, jusqu'aux frontières de la mystique, et au ciel (au paradis) - dans visio beatifica).

Le chemin vers Dieu comme la Bonté-Vérité-Beauté passe donc également par le « louer », l'" honorer » et le « servir ». Saint Ignace écrit dans le Fondement : «L'homme est créé pour louer, honorer et servir Dieu, notre Seigneur, et, par ce moyen, sauver son âme $»^{37}$, ajoutant que " les autres choses qui sont sur la terre sont créées à cause de l'homme et pour l'aider dans la poursuite de la fin que Dieu lui a marquée en le créant $»^{38}$. Les verbes : « louer », « honorer», « servir » expriment la direction fondamentale de l'homme vers Dieu et, bien qu'ils aient des autres significations, se complètent, car, comme le dit W. Królikowski, « louer » signifie : voir et contempler les dons de l'amour de Dieu en nous et autour de nous, se rassasier d'eux (les goûter) et remercier Dieu de ces dons comme le Donneur de tout bien; « honorer » signifie : respecter et réaliser la volonté de Dieu, et « servir » signifie : réaliser la volonté de Dieu, c'est-à-dire entrer dans le plan du salut de toute réalité, prier et faire des bonnes choses pour l'amour de Dieu $^{39}$. La conséquence de cette attitude est la vie quotidienne en harmonie avec soi, avec les autres hommes et avec Dieu ${ }^{40}$.

Selon, par exemple, K. Frejusz, l'amour de Dieu pour l'homme se manifeste dans les créatures ${ }^{41}$. Les créatures peuvent conduire vers Dieu et aussi ne pas conduire à Lui, et c'est pourquoi leur bon choix est si important. Ce choix se lie avec l'indifférence ignatienne (la disposition de la volontét ${ }^{42}$. C'est la question du choix moral et ontologique qui s'oriente vers l'union avec Dieu-Amour. Cette union est le final de la $3^{\mathrm{e}}$ étape du chemin spirituel néoplatonicien ${ }^{43}$. La gloire de Dieu et la propre sanctification de l'homme sont le critère de ce choix ${ }^{44}$. Seulement le péché ne peut pas être accepté dans ce choix et, comme le dit J. Seremak, on ne peut pas être indifférent à une telle réalité ${ }^{45}$. Le bon choix à la réalisation du

${ }^{37}$ Ignacy Loyola, Ćwiczenia duchowne, 23 (trad. J. Sprutta).

${ }^{38}$ Ibidem. Cf. „Exercices spirituels” de saint Ignace de Loyola, trad. du texte espagnol P. Jennesseaux CJ, 1913 (2005), [online] https://livres-mystiques.com/partieTEXTES/Exercices_Ignace/ exercices.html [30.05.2019].

${ }^{39}$ W. Królikowski, Rozwój duchowy. Dynamika „Ćwiczeń duchowych” św. Ignacego Loyoli, Kraków 2013, p. 21. Por. W. Królikowski, Ignacjański Fundament ..., p. 85. Cf. ibidem, pp. 86-87.

${ }^{40}$ W. Królikowski, Rozwój duchowy ..., p. 21.

${ }^{41}$ K. Frejusz, Integralny wymiar pedagogii ..., p. 45.

${ }^{2}$ J. Seremak, Obojętność w „Ćwiczeniach duchowych” [dans :] Droga duchowego uporząkowania i miłości. Podstawowe pojęcia w duchowości ignacjańskiej, red. W. Królikowski, Kraków 2017, pp. 139-151, ici : p. 144.

${ }^{43}$ W. Królikowski, Ignacjański Fundament ..., p. 88.

${ }^{44}$ Ibidem, p. 91.

45 J. Seremak, Obojętność..., p. 143. 
principe magis comme son fondement, c'est-à-dire ce choix est le choix de ce qui est meilleur, plus utile sur le chemin vers Dieu ${ }^{46}$.

Le néoplatonisme montre des vertus comme le sujet de tel choix. Les vertus sont un bien qui mène à l'Absolu. Selon Platon, ce sont les vertus civiques (G. Reale estime que ces vertus se limitent à dominer les passions), chez les néoplatoniciens ce sont les vertus purifiantes (c'est-à-dire les vertus qui libèrent des passions et permettent à l'âme de s'unir avec l'Intellect ; elles sont situées plus haut dans la hiérarchie des vertus que les vertus civiques) ; et les vertus qui rendent l'âme semblable à Dieu (ces vertus sont situées au plus haut dans la hiérarchie des vertus $)^{47}$. Ainsi, la réalisation des vertus dans la vie quotidienne est l'un des chemins néoplatoniciens vers l'Un. Les vertus transforment la vie de l'homme. Seulement l'âme transformée, c'est-à-dire purifiée, peut pleinement connaître la vérité (mais pas l'essence même de Dieu), estimer et réaliser (avec l'engagement) le bien (c'est-à-dire les vertus) et admirer le beau, parce que l'âme transformée, comme extrasensorielle, peut regarder les idées du vrai, du beau et du bien manifestées, plus ou moins, dans la réalité créée ${ }^{48}$. Selon Plotin, la purification de l'âme est la plus importante tâche de la vertu ; cette purification permet à l'âme d'être constamment dans l'unité divine ${ }^{49}$.

\section{Le chemin vers la Vérité (l'étape de l'illumination)}

Le temps passé à Manresa est pour saint Ignace une expérience intensive de la lumière de Dieu. Dieu lui donne cette lumière surtout dans la grande illumination sur la rivière Cardoner $^{50}$. Ignace rappelle cette expérience dans son Autobiographie:

Une fois il se rendait, pour sa dévotion, dans une église qui se trouvait à un peu plus d'un mille de Manrèse je crois qu'elle s'appelle Saint-Paul et le chemin longe la rivière. Il marchait donc, plongé dans ses dévotions, puis il s'assit pour un moment, le visage tourné vers la rivière qui coulait en contrebas. Comme il était assis en cet endroit, les yeux de son entendement commencèrent à s'ouvrir et, sans percevoir aucune vision, il eut l'intelligence et la connaissance de choses nombreuses aussi bien spirituelles que relevant de la foi et de la culture profane et cela avec

${ }^{46}$ Ibidem, p. 144.

47 G. Reale, Historia ..., pp. 591-592.

${ }^{48}$ Cf. T. Stępień, Czystość serca a poznanie prawdy. Pseudo-Dionizy Areopagita i św. Augustyn wobec filozoficznej nauki o czystości], « Warszawskie Studia Teologiczne » 15 (2002), pp. 121-132, ici : p. 124.

${ }^{49}$ P. Hadot, Plotyn albo prostota spojrzenia, Kęty 2004, p. 63.

${ }^{50}$ S.T. Zarzycki, Ćwiczenia ..., p. 179. 
une illumination si grande que toutes ces choses lui paraissaient nouvelles. On ne peut exposer clairement les notions particulières qu'il entendit alors, bien qu'elles eussent été nombreuses, sauf qu'il reçut une grande clarté dans l'entendement ${ }^{51}$.

Il y a ici une dépendance de la vie de saint Ignace d'Antioche : Ignace de Loyola connaît sa vie grâce à la lecture de Flos Sanctorum; chez Ignace d'Antioche (qui suit l'expérience de saint Paul) la croix du Christ illumine, étant au centre de tout. L'illumination comme le don conduit Ignace de Loyola à s'ouvrir à la connaissance profonde ${ }^{52}$.

$2^{\mathrm{e}}$ Semaine des Exercices est la phase de l'illumination (via illuminativa). À cet étape, la plus grande sensibilité du sentiment intérieur complète le travail intellectuel (jusqu'ici dominant). Cette phase concerne également l'aspiration à la perfection chrétienne. Le chemin pré-contemplatif de la $1^{\mathrm{e}}$ Semaine et le chemin contemplatif de la $2^{\mathrm{e}}$ Semaine autrefois interprétés comme l'unique épisode de «l'ascension spirituelle » vers Dieu ont la dimension ascétique dans le passé, puisqu'on les est appelés « la ascèse $»^{53}$. Le chemin de l'illumination est avant le chemin de l'union. C'est nécessaire, car, comme le dit A. Palusińska, l'élévation de l'âme à Dieu et sa théotisation doivent être précédées de la disposition d'accepter la connaissance sur Dieu (révélée dans l'Écriture Sainte, d'où la méditation) et de la disposition de la coopération avec Dieu ${ }^{54}$.

Au sujet de l'illumination comme le chemin vers Dieu, Jean Scot Érigène souligne que l'illumination de l'intellect humain s'accomplie par l'Écriture Sainte et par des créatures qui font la référence à la Sagesse de Dieu dans le monde ${ }^{55}$. La contemplation doit être précédée par l'illumination, car on ne peut pas contempler Dieu sans le connaître. Sans connaître Dieu on ne peut pas Le louer, L'honorer et Le servir. L'illumination couronne la recherche de la vérité et est faite par la Vérité (cf. par exemple l'illuminisme chez Augustin). Jésus-Christ, Dieu incarné, parle de lui-même comme de la Vérité, étant l'image du Dieu le Père. Oint par le Saint-Esprit, Jésus-Christ révèle la vérité sur Dieu le Père et il révèle Dieu le Père comme la Vérité, donc le rapprochement à Dieu par la réflexion (la méditation) ou l'imagination (la contemplation), par l'illumination (interprétée en catégories de la grâce de Dieu), permet de connaître Dieu ou de connaître la Vérité et de participer à cette Vérité. De plus, les Exercices spirituels sont le rapprochement

${ }^{51}$ Ignacy Loyola, Opowieść pielgrzyma. Autobiografia, tłum. M. Bednarz, Kraków 2009, p. 30 ([online] http://voiemystique.free.fr/ignace_de_loyola_autobio_01.htm [30.05.2019]; trad. A. Rocha).

52 Ignacy Loyola, Pisma ..., p. 648.

${ }^{53}$ F. Jalics, Kontemplacja ..., p. 17 (trad. J. Sprutta).

${ }^{54}$ A. Palusińska, Hierarchiczność i partycypacja jako filozoficzne kategorie teorii ikony, Lublin 2017, p. 39.

${ }_{55}$ A. Kijewska, Eriugena, p. 114-115. 
à Jésus-Christ pour Le suivre par la méditation sur ses mystères de sa vie et leur contemplation.

Pseudo-Denys l'Aréopagite écrit à propos de la Parole de Dieu comme la Vérité connue de l'Écriture Sainte: «La Parole divine est plus simple que toute simplicité et elle est indépendante de tout, étant sur-substantiellement au-dessus de tout. Cette Parole est la Vérité simple et réellement existante, qui (comme la connaissance pure et infaillible de toutes choses) se réfère à la foi divine $[\ldots] \gg^{56}$. En dirigeant la pensée vers la vérité interprétée comme transcendentalium et comme l'Absolu (la Vérité), l'homme devient bon, et le bien est identifié (dans les traditions : préchrétienne et chrétienne) avec la beauté. Ainsi, l'atteindre la perfection dans le bien est identifié avec l'atteindre la beauté spirituelle.

La triade initiée par Platon et puis reprise par le néoplatonisme « caractérise » également cette phase du développement spirituel. En outre, la connaissance de Dieu devient nécessaire pour la réalisation de l'attitude suggérée dans le Fondement. Selon Maître Eckhart, « la connaissance est la base et le fondement de chaque être $\aleph^{57}$. L'attitude du Fondement conduit de facto à la theoria chrétienne (vue de Dieu) $^{58}$. À propos de cette théoria, de laquelle l'âme rationnelle est capable ${ }^{59}$, le néoplatonicien Nicolas de Kuza écrit : « La contemplation du Suprême est l'entrée dans la nuit de Dieu et l'entrée dans l'obscurité, qui est née de l'éclat éblouissant ${ }^{60}$, et cette obscurité est (pour Nicolas de Kuza) la nature de Dieu, dont la connaissance est impossible par une créature ${ }^{61}$.

\section{Le chemin vers la Beauté (l'étape de l'union)}

Le chemin basé sur la contemplation est l'étape de l'union, qui correspond à $4^{\mathrm{e}} \mathrm{Se}$ -

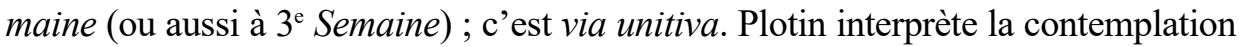
comme l'acceptation de la lumière ${ }^{62}$, et Pseudo-Denys l'Aréopagite écrit sur Jésus-

${ }^{56}$ Pseudo-Dionizy Areopagita, Pisma ..., VII, 4 (trad. J. Sprutta).

${ }^{57}$ Eckhart, Kazanie 71 [dans :] Eckhart, Dzieła wszystkie, thum. W. Szymona, vol. III, Poznań 2014, p. 75 (trad. J. Sprutta).

${ }^{58}$ Cf. J. Skrzypek-Faluszczak, Ocalenie ..., pp. 38, 59, 60. Cf. pp. 210, 212.

59 J. Disse, Metafizyka ..., p. 109.

${ }^{60}$ P. Tendera, Od filozofii światta ..., p. 97 (trad. J. Sprutta).

${ }^{61}$ Ibidem.

${ }^{62}$ A. Kijewska, Neoplatonizm Jana Szkota Eriugeny..., pp. 64-65. Selon Augustin et Bonaventure, l'esprit humain a la lumière qui lui permet de savoir et d'aimer ce qu'il voit comme bon et beau ; cette lumière vient de Dieu. P. Tendera, Od filozofii światta ..., pp. 109-110. Chaque être participe à la bonté et à la beauté. Dieu comme «Un, Bien et Beauté » est la cause de ce qui est beau et bon. Ibidem. Pseudo-Dionizy Areopagita, Pisma ..., IV, 7. 
Christ comme le Rayon dans son traité intitulé Les noms de Dieu ${ }^{63}$. À cet étape, il est le plus radical changement dans la vie spirituelle (dans les Exercices il est délicat et graduel). Ce changement nécessite la confiance totale de l'homme. C'est la phase dans laquelle Dieu prend l'initiative et dans laquelle l'homme se plonge dans le bonheur spirituel, participant à la vision de Dieu. Cette initiative divine est l'acte de l'amour infini, puisque Dieu n'hésite pas à accepter le pécheur dans ses bras et lui permet de contempler son visage ressuscité. Mais premièrement l'homme doit préparer la place pour Dieu en lui-même : « Ce qui est divin peut se retrouver dans ce qui est créé, mais seulement lorsque ce qui est créé fera place au divin en soi $»^{64}$.

La contemplation mystique est le résultat d'un mouvement spirituel et analogique vers l'union avec Dieu, caractérisé par un pur dynamisme ${ }^{65}$. L'union avec Dieu devient possible grâce à l'âme, qui est le don de Dieu à l'homme. Selon Maître Eckhart, cela devient réel à cause de « la petite étincelle » ou du « pic» de l'âme présente dans l'homme ${ }^{66}$. À l'étape de l'union, Dieu donne la contemplation comme la grâce à l'homme. La pensée discursive est abandonnée et aussi le regard intérieur sur son propre développement spirituel, mais cela ne signifie pas le renoncement à l'usage actif de l'attention désormais exclusivement tournée vers Dieu. F. Jalics souligne que si la grâce devient plus forte, alors l'activité humaine ralentie ${ }^{67}$.

Le fait d'atteindre Dieu implique la vie dans la grâce sanctifiante (l'effet de l'étape de la purification) et la connaissance de Dieu (l'effet de l'étape de l'illumination) et de manière de plus en plus parfaite, c'est-à-dire selon le principe magis. Il a le lieu sur « le chemin » d'éliminer des obstacles de soi-même et de notre vie quotidienne, qui sont les obstacles au chemin vers Dieu. Ainsi, il faut choisir seulement des choses qui nous approchent de $\operatorname{Dieu}^{68}$. De nouveau apparait la question de l'indifférence ignatienne évoquant la capacité de choisir des bonnes choses, c'est-à-dire des choses qui « conduisent » vers Dieu. Cette « indifférence », qui signifie l'état de l'équilibre spirituel interne, aide — ensemble avec l'attitude de magis - à atteindre le But final ${ }^{69}$. En conséquence, le Fondement enseigne de situer Dieu en premier lieu, et situer les créatures en leur lieu propre.

${ }^{63}$ Cf. Y. de Andia, Le Bien et l'Être dans les Noms divins de Denys 'Aréopagite [dans :] Being or Good? Metamorphoses of Neoplatonism, ed. A. Kijewska, Lublin 2004, p. 254.

${ }^{64}$ M. Manikowski, Warunki doświadczenia mistycznego..., p. 18-19 (trad. J. Sprutta).

65 J.M. Nieva, "Ejercisios espirituales" y transcendencia en Dionisio Areopagita, « Teología y Vida », 43 (2002), p. 303-309, ici : p. 305.

${ }_{66}$ J. Disse, Metafizyka ..., p. 111.

${ }^{67}$ F. Jalics, Kontemplacja ..., p. 18.

${ }^{68}$ K. Frejusz, Integralny wymiar pedagogii..., p. 45.

${ }^{69}$ W. Królikowski, Rozwój duchowy ..., p. 14. Cf. K. Frejusz, Integralny wymiar pedagogii ..., p. 45. Cf. W. Królikowski, Ignacjański Fundament ..., p. 92. 
Le beau comme transcendentalium correspond au chemin de l'union. Également ici, comme aussi dans la $3^{\text {e }}$ Semaine, la contemplation est l' " unique » méthode de la prière. La perception de la vie de Dieu Incarné permet la participation à ses mystères, qui est basée sur l'échange d'amour entre Dieu et l'homme. La contemplation se réfère à l'imagination (c'est pourquoi elle est la contemplation imaginaire) et à la méditation (la méthode de prière caractéristique pour le Fondement, $1^{\mathrm{e}}$ Semaine et en partie pour $2^{\mathrm{e}}$ Semaine) qui nous mènent vers une réflexion $^{70}$. Dans la contemplation imaginaire, l'image occupe la plus importante place, et dans la méditation cette place est la place du mot. T.M. Gallagher affirme que l'essence des deux méthodes (la contemplation et la méditation) est réduite à la simple intuition exprimée dans la conviction que l'entrée dans le contenu de la parole de Dieu par la réflexion et l'imagination est possible. En outre, ce théologien écrit que grâce à la réflexion ou à l'imagination le cœur de l'homme en prière peut pleinement suivre les mouvements de la grâce ${ }^{71}$. De facto la méditation christocentrique dans les Exercices spirituels inaugure les Exercices, et la contemplation, présente déjà dans la $2^{\mathrm{e}}$ Semaine, est le couronnement des Exercices $^{72}$. Dans la méditation, le travail de la volonté est important de même que le rôle de la raison et de la mémoire, c'est-à-dire le comprendre et le souvenir. Dans une telle contemplation (évangélique, imaginative) il faut aussi souligner le rôle actif des sens : vision, audition, contact et goût, ainsi que de l'intuition. Ce sont les sens intérieurs. Ils apparaissent comme une expérience sensuelle qui décide de la perception spirituelle et permet de voir directement Dieu et sa présence dans le monde ${ }^{73}$.

Dans la contemplation, il y a la soumission à la spirituelle passivité contemplative qui se lie avec l'attitude de la veille intérieure et qui base sur la perception imaginaire des personnages et des événements qui sont l'objet de la contemplation. Grâce à cela, l'image soumise à telle perception devient quasi vivante, elle devient tangible. Ce n'est pas l'image matérielle, bien que l'imagination permette la netteté de telle image ; c'est pourquoi cette image semble même tangible, réelle, proche, et le sujet de la contemplation souvent est présent à cette image. Telle image également souvent rend possible une catharsis spécifique, menant bien sûr à Dieu. Cette image est l'œuvre de l'imagination et n'est pas l'obstacle

${ }^{70}$ Cf. T.M. Gallagher, Medytacja i kontemplacja. Św. Ignacy uczy jak modlac się Pismem Świętym pokochać Jezusa, tłum. M. Chojnacki, Kraków 2015, p. 42.

${ }^{71}$ Ibidem.

72 Cf. H.U. von Balthasar, Medytacja chrześcijańska, tłum. W. Szymona, Poznań 2014, pp. 50 51. Cf. J. Augustyn, Medytacja ignacjańska. Geneza i praktyka, Kraków 2013, pp. 39-50.

${ }^{73}$ M.R. Lootens, Augustyn, p. 88. Cf. P.L. Gavrilyuk, Pseudo-Dionizy Areopagita [dans :] P.L. Gavrilyuk, S. Coakley, Duchowe zmysty. Percepcja Boga w zachodnim chrześcijaństwie, thum. A. Gomola, Kraków 2014, p. 131. 
sur le chemin vers Dieu, car elle aide de venir à Dieu, donc, selon la suggestion contenue dans le Fondement, le rejet de cet image n'est pas nécessaire.

Ignace de Loyola ajoute :

Pour cela, il est nécessaire de nous rendre indifférents à l'égard de tous les objets créés, en tout ce qui est laissé au choix de notre libre arbitre et ne lui est pas défendu ; en sorte que, de notre côté, nous ne voulions pas plus la santé que le maladie, les richesses que la pauvreté, l'honneur que le mépris, une longue vie qu'une vie courte, et ainsi de tout le reste ; désirant et choisissant uniquement ce qui nous conduit plus sûrement à la fin pour laquelle nous sommes créés ${ }^{74}$.

Tel chemin est également connu pour la pensée néoplatonicienne, mais dans le néoplatonisme ce chemin passe par l'art matériel, c'est-à-dire non par l'imagination (l'image imaginée). L'image immatérielle, mais quasi " matérialisant » la réalité soumise comme le sujet à la contemplation, mène au-delà d'elle-même, orientant vers Dieu, ainsi « cachée » dans l'imaginaire cette image a une dimension transcendantale ${ }^{75}$. Il faut passer par la phase de la perception de l'image à un certain moment, et c'est à propos de l'image présente dans l'imagination et de l'image matérielle. La contemplation de l'image matérielle devrait « se brûler [...] dans la vision de l'Invisible et de l'Indicible $»^{76}$. En ce moment, l'image matérielle « cesse d'exister, après avoir épuisé son rôle à conduire au silence de l'Un où la beauté sensuelle n'est pas déjà la valeur, qui doit être créée, car à partir de ce moment, l'âme elle-même est belle et son existence est l'œuvre d'art » ${ }^{77}$. Malgré les similitudes avec la doctrine de Pseudo-Denys l'Aréopagite ou Maître Eckhart, Jésus-Christ crucifié est beau pour saint Ignace de Loyola et les contemplations sont très réalistes, et compositio loci, le kénose et l'incarnation de Dieu, l'amour en actes, l'amour de l'Eglise et l'amour dans l'Eglise (cf. sentire cum Ecclesia chez Ignace) sont importants.

L'image immatérielle (imaginée), et, dans le néoplatonisme, également l'image matérielle mènent vers la Beauté. Le beau qui se manifeste dans les deux types de l'image fait référence à la Beauté absolue, qui est « le début et la fin de la beauté [tout - J.S.] $»^{78}$. L'âme désire cette Beauté, c'est pourquoi Plotin l'encourage à dépasser les limites de la matière, à dépasser ce qui est moins formel, croyant que ça encore plus (magis) va augmenter son (c'est-à-dire de l'âme)

${ }^{74}$ Exercices spirituels..., 23 [30.05.2019].

${ }^{75}$ K. Sztuka, Wyobraźnia ..., p. 234.

${ }^{76}$ G. Ravasi, M.I. Rupnik, Fascynacja pięknem. Między Biblia a teologia, Kraków 2013, p. 41 (trad. J. Sprutta).

${ }^{77}$ Ibidem, p. 42 (trad. J. Sprutta).

78 Plotyn, Enneady, thum. A. Krokiewicz, Warszawa 2004, VI, 7 (trad. J. Sprutta). 
désir « esthétique $»^{79}$. Néanmoins, l'élément divin est également présent dans l'élément matériel. De plus, le Créateur lui-même s'appelle l'Artiste (Artifex).

La présence mentionnée fait que l'image peut approcher de Dieu même si elle peut également éloigner de Dieu : l'image matérielle et l'image imaginée. Cette vérité concerne aussi par exemple la nature qui est un chef d'oeuvre de Dieu-Créateur. Et de nouveau l'homme doit choisir, et de nouveau il doit être « indifférent» (« l'indifférence» ignatienne). La connaissance de Dieu par la réalité créée conduit à la contemplation. Cette connaissance est possible, parce que se sont les vestigia Dei (cf. : chez saint Augustin) qui se rendent présentés dans cette réalité. T. Stępień écrit : « Nous pouvons Le [Dieu - J.S.] connaître seulement par ce qui provient de Lui. Chaque créature porte en soi une trace de l'action de Dieu. Cette trace est d'autant plus grande que la création est parfaite $»^{80}$.

Néanmoins, la connaissance de Dieu n'est pas encore la contemplation, bien qu'elle puisse y conduire. Du reste la beauté, la bonté et la vérité s'étendent audelà d'elles-mêmes : à l'Absolu, dont transcendentalia sont les attributs et les noms $^{81}$. Ces transcendentaux se rapportent à Dieu Incarné qui a révélé le visage miséricordieux du Père dans l'Esprit Saint sur la terre, et qui le fait toujours, par exemple à partir du Fondement, dans les Exercices spirituels christocentriques. Dans ce contexte, J.P. Strumiłowski écrit que seulement Jésus est un signe qui ne montre pas au-delà de lui-même, mais directement lui-même, car Il est « désigné » par lui-même ${ }^{82}$ : autour de ce "signe » Ignace de Loyola a placé le Fondement et les quatre Semaines des Exercices.

La pensée d'un retraitant ne s'arrête pas à la forme de la méditation ou de la contemplation, car les étapes du chemin spirituel qu'il passe le perfectionnent, lui donnant une maturité intérieure, harmonieuse, spirituelle et relationnelle. Dans ce contexte, le recueillant décide de réaliser l'attitude du Fondement dans sa vie quotidienne, déjà au-delà des retraites. Cet attitude est : " louer », " honorer » Dieu et « servir » à Dieu, c'est-à-dire : faire tout cela dans l'esprit du magis et avec « l'indifférence » ignatienne.

\footnotetext{
79 Ibidem.

80 T. Stępień, Pseudo-Dionizy Areopagita ..., p. 95 (trad. J. Sprutta).

81 Cf. J.P. Strumiłowski, Piękno zbawi świat?, Kraków 2016, p. 140. Cf. pp. 148-149.

82 Ibidem, p. 140.
} 


\section{Neoplatonic dimension of the Ignatian Foundation (in the context of the entire Spiritual Exercises of St. Ignatius of Loyola)}

\section{Summary}

God is the foundation and goal of man. The way to God, from the state of disgrace to a happy relationship with God, is also the "foundation" of the Spiritual Exercises of St. Ignatius of Loyola, including the Foundation. In the Foundation there is a Neoplatonic way to God as absolute GoodTruth-Beauty. The spiritual way, continued in Weeks of the Ignatian retreat, includes the stages of purification, enlightenment and unification. This way is thus also an existential principle present in Christian Neoplatonism, having its reception in all cycle of Ignatian Exercises. The article to concern the relationship between the theology of the Foundation and Christian Neoplatonism, with reference to the whole of the Spiritual Exercises of St. Ignatius of Loyola.

\section{Keywords}

Ignatian spirituality, Spiritual Exercises, Ignatian Foundation, Christian Neoplatonism, spiritual way (purification, enlightenment, unification), God as the Good-Truth-Beauty

\section{Neoplatoński wymiar Fundamentu ignacjańskiego (w kontekście caloksztaltu Ćwiczeń duchownych świętego Ignacego Loyoli)}

\section{Streszczenie}

Bóg jest fundamentem i celem człowieka. Droga do Boga, od stanu niełaski do uszczęśliwiającej więzi z Bogiem, stanowi także osnowę ignacjańskich Ćwiczeń duchownych, w tym inicjującego je Fundamentu. W Fundamencie kryje się neoplatońska droga ku absolutnemu Dobru-PrawdziePięknu, czyli ku Bogu. Duchowa droga, kontynuowana w Tygodniach rekolekcji ignacjańskich, obejmuje etapy: oczyszczenia, oświecenia i zjednoczenia. Droga ta jest zatem również egzystencjalną zasadą obecną w chrześcijańskim neoplatonizmie, mającą swoją recepcję w całym cyklu ignacjańskich Ćwiczeń. Artykuł dotyczy związku między teologią Fundamentu a chrześcijańskim neoplatonizmem, w odniesieniu do całokształtu Ćwiczeń duchownych.

\section{Słowa kluczowe}

duchowość ignacjańska, Ćwiczenia duchowne, Fundament ignacjański, neoplatonizm chrześcijański, droga duchowa (oczyszczenie, oświecenie, zjednoczenie), Bóg jako Dobro-Prawda-Piękno 


\section{Bibliographie}

Andia de Y., Le Bien et l'Être dans les Noms divins de Denys l'Aréopagite [dans :] Being or Good? Metamorphoses of Neoplatonism, éd. A. Kijewska, Lublin 2004, pp. 253279.

Augustyn J., „Ćwiczenia duchowne” w praktyce. Uwagi odnoszace się do udzielania i odprawiania „Ćwiczeń duchownych” "św. Ignacego Loyoli z petnym milczeniem i indywidualnym prowadzeniem, Kraków 2013.

Augustyn J., Medytacja ignacjańska. Geneza i praktyka, Kraków 2013.

Balthasar von U.H., Medytacja chrześcijańska, tłum. W. Szymona, Poznań 2014.

Bednarz M., Całościowa wizja „Ćwiczeń duchownych” św. Ignacego [dans :] Co zabrać ze soba? Po Fundamencie „Ćwiczeń duchownych”, red. J. Augustyn, Kraków 2012, pp. 13-28.

Disse J., Metafizyka. Od Platona do Hegla, thum. A. Węgrzecki, L. Kusak, Kraków 2005. Dziennik duchowy św. Ignacego, tłum. i oprac. M. Bednarz, vol. I, Kraków 1968.

Eckhart, Dzieła wszystkie, tłum. W. Szymona, vol. III, Poznań 2014.

"Exercices spirituels" de saint Ignace de Loyola, trad. du texte espagnol P. Jennesseaux CJ, 1913 (2005), https://livres-mystiques.com/partieTEXTES/Exercices_Ignace/ exercices.html [accès 30.05.2019].

Frejusz K., Integralny wymiar pedagogii „Ćwiczeń duchowych” św. Ignacego Loyoli, «Studia Bobolanum» 28 (2017), 2, pp. 33-59.

Gallagher M.T., Medytacja i kontemplacja. Św. Ignacy uczy jak modlac się Pismem Świętym pokochać Jezusa, tłum. M. Chojnacki, Kraków 2015.

Gavrilyuk L.P., Pseudo-Dionizy Areopagita [dans :] P.L. Gavrilyuk, S. Coakley, Duchowe zmysty. Percepcja Boga w zachodnim chrześcijaństwie, thum. A. Gomola, Kraków 2014, pp. 117-138.

Gogacz M., Szkice o kulturze, Kraków-Warszawa/Struga 1985.

Hadot P., Plotyn albo prostota spojrzenia, Kęty 2004.

Ignacy Loyola, Ćwiczenia duchowne, tłum. J. Ożóg, Kraków 1997.

Ignacy Loyola, Opowieść pielgrzyma. Autobiografia, tłum. M. Bednarz, Kraków 2009.

Ignacy Loyola, Pisma wybrane, thum. i oprac. M. Bednarz, vol. I, Kraków 1968.

Jalics F., Kontemplacja. Wprowadzenie do modlitwy uważności, tłum. J. Bolewski, Kraków 2017.

Kijewska A., Eriugena, Warszawa 2005.

Kijewska A., Neoplatonizm Jana Szkota Eriugeny. Podmiotowe warunki doświadczenia mistycznego w tradycji neoplatońskiej, Lublin 1994.

Królikowski W., Ignacjański Fundament. Sztuka korzystania ze stworzeń [dans :] Droga duchowego uporzadkowania i mitości. Podstawowe pojęcia w duchowości ignacjańskiej, red. W. Królikowski, Kraków 2017, pp. 83-95.

Królikowski W., Rozwój duchowy. Dynamika „Ćwiczeń duchowych” św. Ignacego Loyoli, Kraków 2013. 
Kunzmann P., Metamorfozy neoplatonizmu, « Poznańskie Studia Teologiczne » vol. XXII (2008), pp. 203-214.

Lootens R.M., Augustyn [dans :] P.L. Gavrilyuk, S. Coakley, Duchowe zmysty. Percepcja Boga w zachodnim chrześcijaństwie, tłum. A. Gomola, Kraków 2014, pp. 81-98.

Manikowski M., Warunki doświadczenia mistycznego wedtug Pseudo-Dionizego Areopagity. Ujęcie filozoficzne, « Filozofia Chrześcijańska » 15 (2018), 3, pp. 9-29.

Misiurek J., Zarys historii duchowości chrześcijańskiej, Lublin 1992.

Mrozek S., Rekolekcje w domu ze św. Ignacym Loyola, Kraków 2012.

Nieva M.J., "Ejercisios espirituales” y transcendencia en Dionisio Areopagita, « Teología y Vida » vol. XLIII (2002), pp. 303-309.

O’Brien K., „Ćwiczenia duchowe”. Przewodnik po przygodzie życia, tłum. G. Gomola, A. Gomola, Kraków 2016.

Palusińska A., Hierarchiczność i partycypacja jako filozoficzne kategorie teorii ikony, Lublin 2017.

Plotyn, Enneady, tłum. A. Krokiewicz, Warszawa 2004.

Pseudo-Dionizy Areopagita, Pisma teologiczne, tłum. M. Dzielska, Kraków 2005.

Ravasi G., Rupnik I.M., Fascynacja pięknem. Między Biblia a teologia, tłum. B. Żurowska, Kraków 2013.

Reale G., Historia filozofii starożytnej. Szkoły epoki Cesarstwa, tłum. E.I. Zieliński, vol. IV, Lublin 1999.

Scruton R., Piękno, tłum. S. Krawczyk, A. Rejniak-Majewska, Łódź 2018.

Seremak J., Obojętność w „Ćwiczeniach duchowych” [dans :] Droga duchowego uporządkowania i miłości. Podstawowe pojęcia w duchowości ignacjańskiej, red. W. Królikowski, Kraków 2017, pp. 139-151.

Skrzypek-Faluszczak J., Ocalenie od zła w filozofii Platona, Kraków 2010.

Stępień T., Czystość serca a poznanie prawdy. Pseudo-Dionizy Areopagita i św. Augustyn wobec filozoficznej nauki o czystości, « Warszawskie Studia Teologiczne » vol. XV (2002), pp. 121-132.

Stępień T., Pseudo-Dionizy Areopagita. Chrześcijanin i platonik, Warszawa 2010.

Strumiłowski P.J., Neoplatońska idea wyjścia i powrotu jako strukturalna zasada wyrażania prawd teologicznych $i$ jej dekonstrukcyjna reinterpretacja, « Teologia w Polsce » 10 (2016), 2, pp. 129-140.

Strumiłowski P.J., Piękno zbawi świat?, Kraków 2016.

Sztuka K., Wyobraźnia a rozwój duchowy, Kraków 2010.

Tatarkiewicz W., O doskonatości, Lublin 1991.

Tendera P., Od filozofii światta do sztuki światta, Kraków 2014.

Zarzycki T.S., Ćwiczenia duchowne [dans :] Leksykon duchowości katolickiej, red. M. Chmielowski, Lublin-Kraków 2002, pp. 179-182. 